\title{
Estimation of Local Movement of Ovarian Follicle for Infertility Treatment
}

\author{
Yoshiharu Koya ${ }^{1}$, Tomomoto Ishikawa ${ }^{2}$, Hidehiko Matsubayashi ${ }^{3}$, Yutaka Hata ${ }^{4}$ \\ ${ }^{1}$ Department of Electronic Engineering Kobe City College of Technology \\ 8-3, Gakuen higashimachi, Nishiku, Kobe, Hyogo 651-2194, Japan \\ E-mail:koya@kobe-kosen.ac.jp \\ ${ }^{2}$ Ishikawa Hospital , ${ }^{3}$ Reproduction Clinic Osaka \\ ${ }^{4}$ Dean \& Professor, Graduate School of Simulation Studies, University of Hyogo, Japan
}

\begin{abstract}
In recent years, more and more people have been getting married at a later age, and this trend is only going to increase in the near future. As a result, many infertility treatments have been researched upon and developed. One such treatment is in-vitro fertilization, wherein a follicle is directly absorbed from the ovary of the woman and impregnated with a sperm cell under a microscope. Of the total number of follicles in a woman's body, $20-30 \%$ are usually observed to contain vacuoles, which do not stimulate vitelline growth. As such, in most cases, one cannot judge whether a follicle has an ovum or a vacuole unless we examine it under a microscope. This study proposes a method capable of distinguishing follicles on the basis of local movement.
\end{abstract}

\section{Introduction}

The infertility rate in Japan is presently reported at $15 \%$ implying that one out of every three couples is infertile. Of all the infertile couples in Japan, infertility in women accounts for $50 \%$ of the cases. $30 \%$ of the cases report infertility in men. In the remaining $20 \%$ of the cases, the causes of infertility are yet unknown. Therefore, many infertility treatments have been at the forefront of medical research and development in the country in recent years. Some of these treatments include artificial fertilization, in-vitro fertilization, and micro-fertilization. This paper focuses on in-vitro fertilization and micro-fertilization. Both, in-vitro as well as micro-fertilization are based on the extraction of an ovum from the body of woman. From a female body, an ovum may be extracted from the follicles. In order to extract a follicle from a woman's body, she is pricked with a needle, and 
the follicle is sucked out of the body. During follicle extraction, the woman experiences a state of acute pain.

However, all follicles may not always include an ovum. It is indeed difficult to judge whether a follicle contains an ovum or a vacuole unless the follicle is extracted, examined, and checked under a microscope. The presence of a vacuole implies a follicle without an ovum.

There is, therefore, a need for a method capable of determining whether a follicle contains an ovum or vacuole, prior to it being extracted from the body of a woman. A method that uses ultrasound images has previously been proposed to determine whether a follicle contains an ovum or a vacuole. However, the method was not very reliable, as its detection algorithm was exclusively based on the brightness information contained within the images, and brightness levels of the image, in turn, were subject to change based on imaging conditions that varied from time to time. The proposed study estimates local movement of ovarian follicles to determine the presence of an ovum within a follicle.

\section{Image overview}

The procedure performed by the physician to extract a follicle is depicted in Figure 1. The parts marked red represent a follicle that has been extracted.

The figure depicts the area around the cervix. The sac-like form connected to it is called the ovary. A follicle is present inside and is extracted from this ovarian part. In general, a follicle may contain an ovum or a vacuole. Figure 2 depicts an ultrasound image of the schematic extraction process depicted in figure 1 .

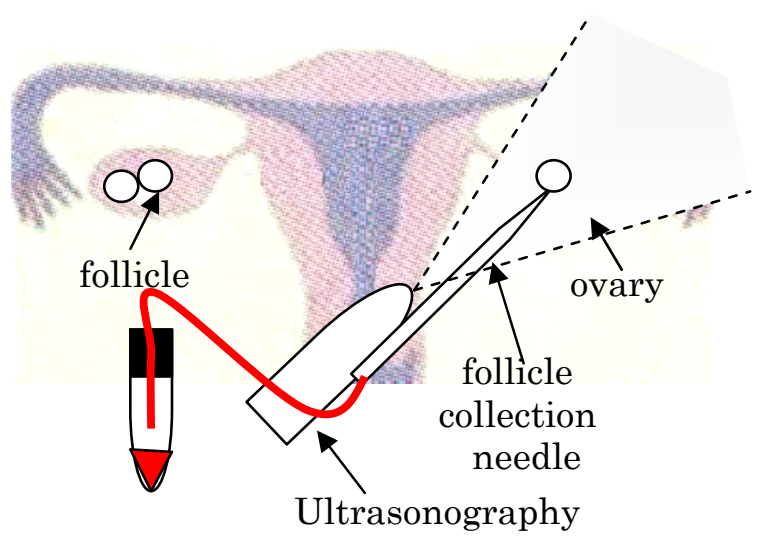

Fig. 1 Illustration of the uterus

During follicle extraction, it is necessary to first extract a follicular edge prior to extraction of the follicle part that comprises the target domain. Some methods for edge extraction have already been suggested. For the sake of conciseness, the authors have omitted details edge extraction in this paper.

Figure 3 shows images of follicles with and without an ovum. The two images must be compared in order to identify the follicle that contains an ovum and the one that does not. However, there is no big difference in the images shown in figure 3 owing to the following reasons.

1) The size of the ovum is very small. The follicle measures over $10 \mathrm{~mm}$ in size, while the size of ovum is only $100 \mu \mathrm{m}$. In other words, the ovum is one-hundredth 
the size of a follicle.

2) The difference in brightness is very small when the follicle contains an ovum.

3) An ovum may not be included in this slice image. In other words, there is a possibility of the ovum having exited the follicle.

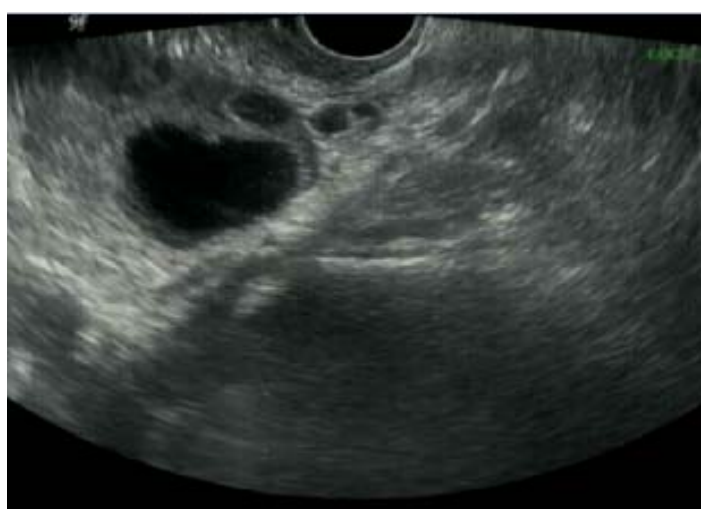

Fig. 2 Ultrasound image of an ovary

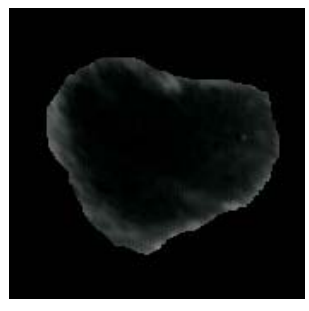

(a)

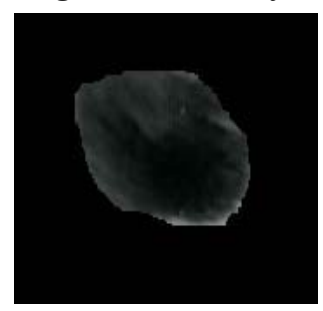

(b) (a) Follicle without ovum (b) Follicle with ovum

Fig. 3 Follicle images

If ultrasound imaging is used, the only information one has to work with is the image brightness, which is not reliable. However, we guessed that the ovum is tense to be going to protect itself. Therefore, in this study, the authors utilized difference in brightness or contrast to estimate the local movement in follicles. Furthermore, if an ovum exists inside a follicle, it must be captured in at least one slice image. As such, multiple slice images of a single follicle, obtained from different angles and sections, were examined in this study.

\section{Principle of estimation of local movement}

An image of the target follicle used in this study is depicted in figure 4 . The image has a resolution of $1280 \times 1024$ pixels along with 256 grey levels. All black domains depicted in the figure represent follicles. Some of these follicles may even contain vacuoles. In the analysis that follows, attention is focussed on the follicle marked by the red box in figure 4 . The follicle was examined using a microscope and found to contain an ovum. As such, an ovum must be captured in at least one slice image.

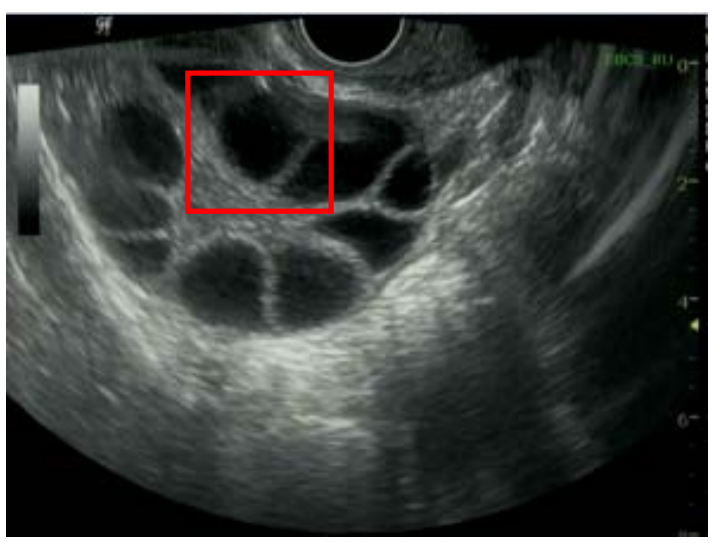

Fig. 4 Target image

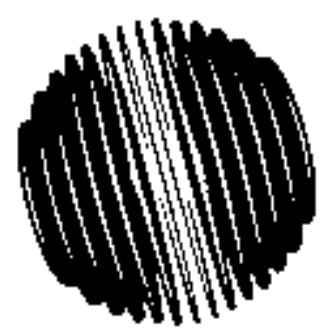

Fig. 5 Illustration of the slice image of a follicle 


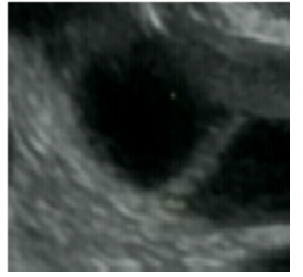

(a)

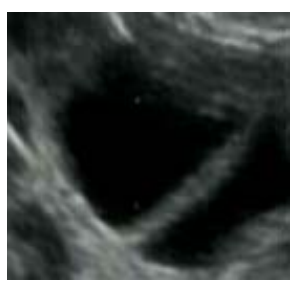

(c)

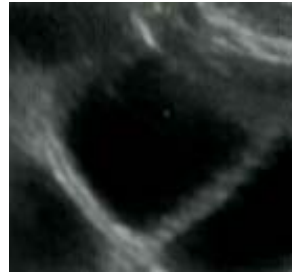

(b)

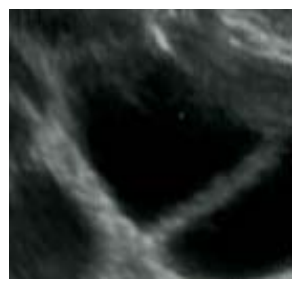

(d)
Fig. 6 Follicle image per one second

The follicles have a round, spherical form, and different sliced images of a follicle are depicted in figure 5. It is understood from the figure that the size of each sliced image is different. Four such sliced images are depicted in figure 6. All four sliced images in the figure are obtained from the same follicle. However, they have not been captured at the same distance from each other, because these images have been manually extracted.

\subsection{Standardization of an Image}

The form of the extracted follicle was standardized, as shown in figure 7 and mathematically described in equation (1).

Because, it is easy to estimate movement amount between a slice image and another slice image. The reason is that it is difference the size of follicle and the length and/or form of edge each slice image.

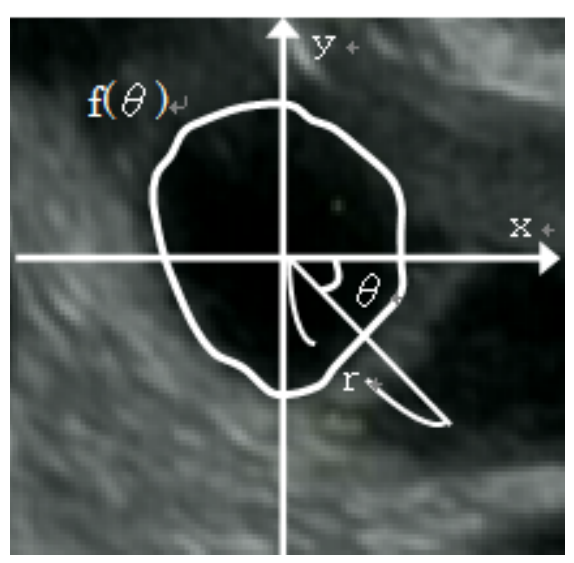

Fig. 7 Coordinate of follicle (Enlarged image of figure 6(a))

$$
\left\{\begin{array}{c}
\hat{r}=p(r, \theta)=\frac{R}{f(\theta)} r \\
\hat{\theta}=q(r, \theta)=\theta
\end{array} \quad \ldots\right.
$$

The parameters $r$ and $\theta$ represent the original coordinate system, while $\hat{r}$ and $\hat{\theta}$ belong to the new or modified coordinate system to which the follicle is being transformed. $\mathrm{R}$ is the radius of the standardized image and $f(\theta)$ is the border of the follicle. A standardized sliced image, obtained using equation (1), is depicted in figure 8 .

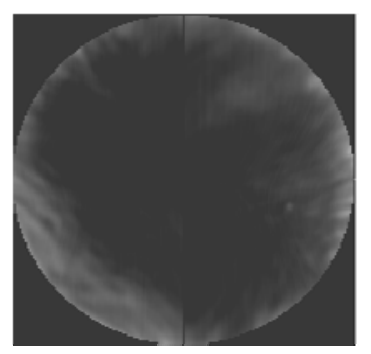

Fig. 8 Standardized form of a follicle

\subsection{Movement Estimation on Standardization Image}

Using equation (2), local and relative movements of the follicle can be estimated.

The movement amount of the new 
coordinate system is estimated using the optical flow shown in equation (2).

The flow velocity $\left(u_{\hat{r}}, u_{\hat{\theta}}\right)$ cannot be computed locally without introducing additional constraints. Therefore, we estimated the flow using spatial local optimization.

$$
\frac{\partial \mathrm{I}}{\partial \hat{\mathrm{r}}} \mathrm{u}_{\widehat{\mathrm{r}}}+\frac{\partial \mathrm{I}}{\partial \widehat{\theta}} \mathrm{u}_{\widehat{\theta}}+\frac{\partial \mathrm{I}}{\partial \mathrm{t}}=0
$$

(note: $\mathrm{I}(\hat{r}, \hat{\theta})$ is brightness of the image.)

\subsection{Change of Movement Amount from} Standardization coordinate to Original coordinate

We have to change the movement amount from the movement of the standardization coordinate to that of the original coordinate. We defined the movement amount of the original coordinate system as $\mathrm{u}_{\mathrm{r}}, \mathrm{u}_{\theta}$, and the movement amount of the new coordinate system as $u_{\hat{r}}, u_{\hat{\theta}}$. Therefore, we use equation (3). $u_{r}$ and $u_{\theta}$ are the sum of the Jacobi matrix, which represents the coordinate transformation and movement amount of the new coordinate system.

$\left[\begin{array}{l}\mathrm{u}_{\mathrm{r}} \\ \mathrm{u}_{\theta}\end{array}\right]=\left[\begin{array}{ll}\frac{\partial \mathrm{p}}{\partial \hat{\mathrm{r}}} & \frac{\partial \mathrm{p}}{\partial \widehat{\theta}} \\ \frac{\partial \mathrm{q}}{\partial \hat{\mathrm{r}}} & \frac{\partial \mathrm{q}}{\partial \widehat{\theta}}\end{array}\right]\left[\begin{array}{l}\mathrm{u}_{\widehat{r}} \\ \mathrm{u}_{\widehat{\theta}}\end{array}\right]+\left[\begin{array}{l}\frac{\partial \mathrm{p}}{\partial \mathrm{t}} \\ \frac{\partial \mathrm{q}}{\partial \mathrm{t}}\end{array}\right]$

The second term on the right of the equality in equation (3) represents a change in the local and relative as well as global movements. Because, this second term represent changing of border, the local and relative movements can be estimated by subtracting this term from the equation.

\subsection{Follicle and Ovum Overview}

Figure 9 demonstrates an illustration of a sliced follicle image.

As can be seen, the size of the sliced image in figure 9(a) is half the size of that in figure 9(b). But, the black circle (ovum) is of the same size. When the standardized image is prepared, both follicles are brought to the same size; i.e., the size of the image in figure 9(a) is doubled to match the size of the follicle in figure 9(b). This implies that the size of the ovum is also doubled. As previously mentioned, the local and relative movements of the follicle can be estimated using equation (3).
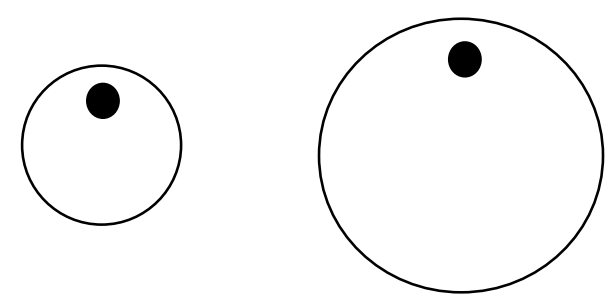

(a) Small sliced image (b)Big sliced image

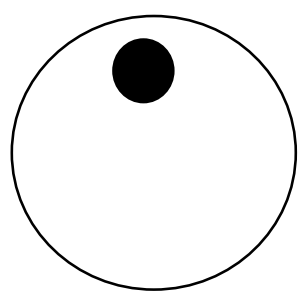

(c)

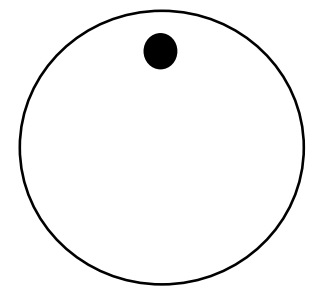

(d) (c) Standardized image of (a)

(d) Standardized image of (b)

Fig. 9 Illustration of follicle sliced images 
For example, in case of figure 9, equation

(3) becomes equation (4).

$$
\left[\begin{array}{l}
\mathrm{u}_{\mathrm{r}} \\
\mathrm{u}_{\theta}
\end{array}\right]=\left[\begin{array}{cc}
\frac{\mathrm{f}(\theta)}{\mathrm{R}} & 0 \\
0 & 1
\end{array}\right]\left[\begin{array}{c}
\mathrm{u}_{\widehat{\mathrm{r}}} \\
\mathrm{u}_{\widehat{\theta}}
\end{array}\right]+\left[\begin{array}{c}
\frac{\partial \mathrm{p}}{\partial \mathrm{t}} \\
0
\end{array}\right]
$$

Using this technique, an outward movement of the follicle was observed in the black domain (figure 4).

This magnitude of the movement is of the same order as the radius of the sliced follicle, as the radius has been doubled. At the same time, the global movement of the follicle can be estimated by not subtracting the second term on the right side of equation (3). In this case, the value of the second term is of the same order as the radius of the sliced follicle. The global movement is, thus, found to be zero. It can, therefore, be inferred that the black domain does not transform.

\subsection{Display of Estimated Movement} Amount

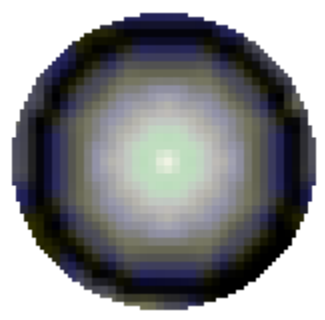

(a)

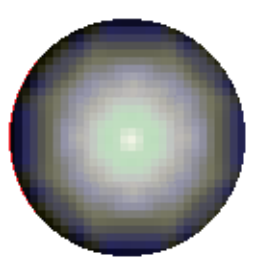

(b) (a) Gaussian Distribution

(b) Image of a part of (a)

Fig. 10 Image of the Gaussian distribution

We show another pattern in figure 10 . These images have the same brightness distribution, but figure 10(b) is part of figure 10(a). Therefore, we estimate the local and relative optical flows, which are expanded. We show the estimation result in figure 11.

From figure 11, we understood that the local and relative optical flows were observed as outwardly directed movements. Moreover, it is hardly a rotation movement. Therefore, we got a good result.

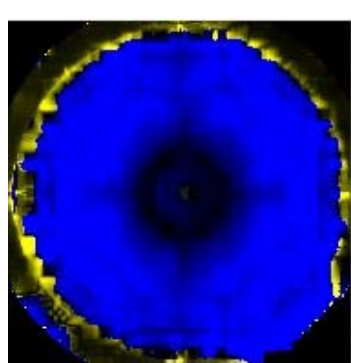

(a)

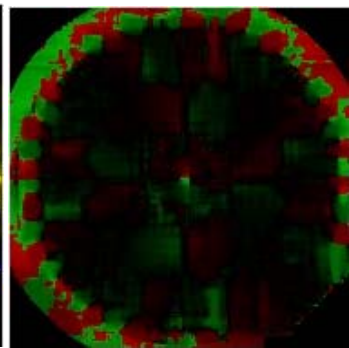

(b)

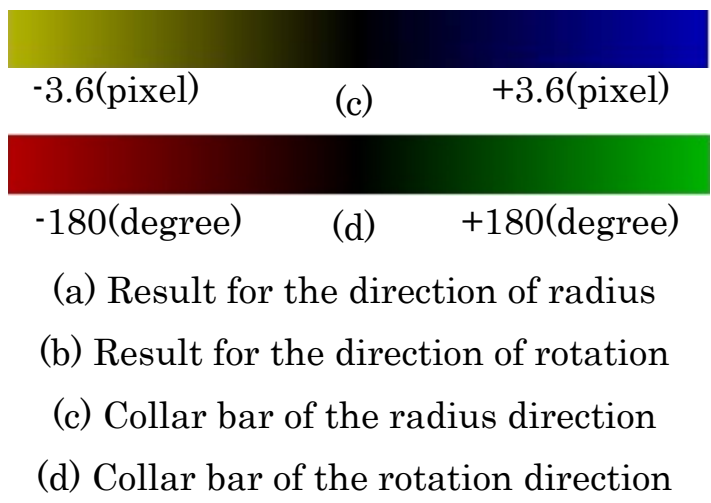

Fig. 11 Results for local and relative flows

\subsection{Adaptation to Follicle}

An estimation of local movement inside the follicle was also performed using the proposed method. This can, however, be done only if the target follicle contains an ovum. The result obtained through application of this method inside the follicle is depicted in figure 12. As a result, 
it is the domain where there may be an ovum because the movement of the yellow domain indicates that it is different from the circumference.

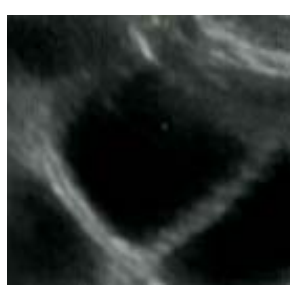

(a)

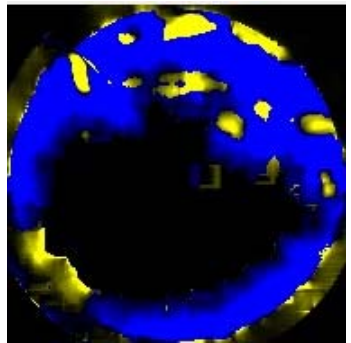

(c)

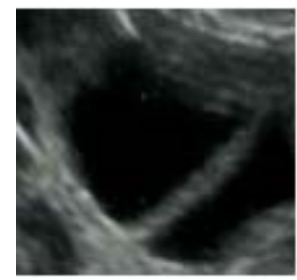

(b)

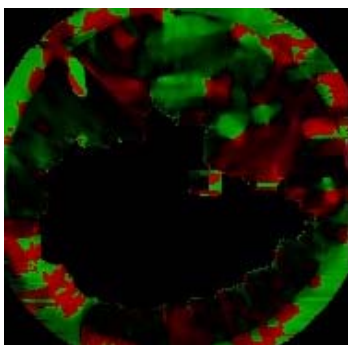

(d) (a) and (b) are the original images, (c) and

(d) are the results of the estimation

Fig. 12 Results for local and relative movements

\section{Conclusion}

In this study, a method capable of identifying follicles that contain ovum prior to they being extracted from a woman's body is proposed. The method is based on the estimation of local and relative movements of the follicle.

We would like to estimate the difference in movement when compared with other areas.

Henceforth, the authors propose to make use of multiple sliced images of the follicle in the analysis and movement estimation thereby facilitating a more accurate estimation of the presence of an ovum or vacuole inside a follicle.

\section{Acknowledgement}

This work was supported in part by a Grant-in-Aid from Hyogo COE Program Promotion Project and Terumo Foundation for Life Sciences and Arts.

\section{Reference}

[1] Yoshiharu Koya, Tomomoto Ishikawa, Hidehiko Matsubayashi, Yutaka Hata "Identification of Ovarian Follicles for Infertility Treatment", Proceedings of the 48th ISCIE International Symposium on Stochastic Systems Theory and Its Applications, pp.93-97 (2017)

[2]Hidehiko Matsubayashi "Supporting Fertility", Vol.2 p.5 Reproduction Clinic Osaka (2016)

[3] Yoshiharu Koya, Masashige Hori, Isao Mizoshiri: "Border Detection of Echocardiogram using Projection Operator", T.IEEJ Japan, Vol.120-C No.8/9 pp.1236-1241 (2000-8) (in Japanese)

[4]Yoshiharu Koya, Isao Mizoshiri " Determination of the Center of Left Ventricule and border detection on Short Axis Image of Echocardiogram ", T.IEEJ Japan, Vol.123-C No.8, pp.1387-1392 (2003-8) (in Japanese)

[5] Yoshiharu Koya, Isao Mizoshiri: " Border detection on Common Carotid Artery using Gauss-Markov Estimation", T.IEEJ Japan, Vol.124-C No.10,pp.2051-2056 (2004-10) （in 
Japanese)

[6] Isao Mizoshiri, Yoshiharu Koya, Takahiro Yamada: " Local and Relative Optical Flow Estimation", T.IEEJ Japan, Vol.118-C No.3,pp.339-345 (1998-3) (in Japanese) 